\title{
ABSOLUTELY CONTINUOUS SPECTRUM OF STARK TYPE OPERATORS
}

\author{
A. A. POZHARSKI
}

Abstract. Some new conditions are obtained for the absolutely continuous spectrum of a Stark operator to fill the entire real line.

\section{$\S 1$. INTRODUCTION}

We consider one-dimensional Stark operators

$$
H=-\frac{d^{2}}{d x^{2}}-x+q(x)
$$

in $L_{2}\left(\mathbb{R}_{+}\right)$with domain

$$
\operatorname{Dom}(H)=\left\{\varphi: \begin{array}{l}
\varphi^{\prime} \text { is absolutely continuous }, \varphi(0)=0, \\
\operatorname{supp} \varphi \text { is compact },-\varphi^{\prime \prime}+q \varphi \in L_{2}\left(R_{+}\right)
\end{array}\right\} .
$$

We assume that the potential $q$ satisfies the inequality

$$
\sup _{x \geq 0} \int_{0}^{1}|q(x+t)| d t<\infty .
$$

The operator $H$ is essentially selfadjoint. For periodic potentials $q$, this fact was proved in [5. For potentials $q$ satisfying (1.1), the proof is similar. We denote by $H_{d}$ the closure of $H$. An extensive literature devoted to the Stark operator can largely be found in the book [6] and the preprint 8 .

It is well known that, for $q=0$, the spectrum of $H_{d}$ is purely absolutely continuous and fills the entire real line. In the paper $\left[8\right.$, the operator $H$ in $L_{2}(\mathbb{R})$ with an appropriate domain was studied, and conditions on the potential $q$ under which the absolutely continuous component of the spectrum of $H$ fills the entire real line, as well as conditions under which the spectrum of $H$ is purely absolutely continuous, were obtained. There, the authors imposed either smoothness conditions on the potential $q$, or some conditions on the rate of decay at infinity. It was also shown that the results of [8] are optimal in each of the two classes. As an example, we present two of the results proved in 8 .

Theorem 1.1. Suppose the potential $q(x)$ is locally integrable and $q\left(x^{2}\right) \in L_{p}(\mathbb{R})$ for some $1 \leq p<2$. Then the absolutely continuous component of the spectrum of the operator $H$ fills the entire real line.

Theorem 1.2. Suppose the potential $q(x)$ satisfies the estimate

$$
\sup _{x}|q(x)|+\sup _{x, y} \frac{|q(x)-q(y)|}{|x-y|^{\alpha}}<\infty
$$

2000 Mathematics Subject Classification. Primary 34L40.

Key words and phrases. Stark operator, spectrum, Weyl function, $B F$-type estimates.

Supported by RFBR (grant nos. 05-01-01076 and 05-01-02944). 
for some $\alpha>1 / 2$. Then the absolutely continuous component of the spectrum of the operator $H$ fills the entire real line.

In the recent paper [12, the absolutely continuous spectrum of $H$ in $L_{2}(\mathbb{R})$ was also studied. We state one of the main results of [12] in a somewhat simpler form.

Theorem 1.3. Suppose the potential $q(x)$ satisfies (1.1) and

$$
\int_{1}^{\infty} \frac{1}{\sqrt{x}} e^{2 \sqrt{x}}\left|\int_{x}^{\infty} \frac{q(y)}{\sqrt{y}} e^{\frac{2 i}{3} y^{3 / 2}-\sqrt{y}} d y\right|^{2} d x<\infty .
$$

Then the absolutely continuous component of the spectrum of the operator $H$ fills the entire real line.

In the present paper, our goal is to prove Theorems 1.4 and 1.6 stated below. We introduce the following notation:

$$
\begin{aligned}
& c_{n}=\int_{0}^{1} q(n+t) e^{2 i \sqrt{n} t} d t, \quad \omega_{n}=\sup _{x \in[0,1]}\left|\int_{0}^{x} q(n+t) e^{2 i \sqrt{n} t} d t\right|, \\
& \hat{\delta}_{n}=\sum_{p=1}^{n-1}\left[\sqrt{p}+\frac{1}{4 \sqrt{p}}\left(1-2 \int_{0}^{1} q(p+t) d t\right)\right], \quad n \in \mathbb{N} .
\end{aligned}
$$

Theorem 1.4. Suppose

$$
\begin{aligned}
& \sum_{n=1}^{\infty} \frac{\left|c_{n}\right|}{\sqrt{n}} e^{-2 \sqrt{n}}\left|\sum_{k=1}^{n-1} \frac{c_{k}}{\sqrt{k}} e^{2 \sqrt{k}+2 i \hat{\delta}_{k}}\right|<\infty, \\
& \sum_{n=1}^{\infty} \frac{\omega_{n}}{n}<\infty, \quad \sup _{n \geq 0} \int_{0}^{1}|q(n+t)| d t<\infty .
\end{aligned}
$$

Then the absolutely continuous component of the spectrum of the operator $H_{d}$ fills the entire real line.

Corollary 1.5. Let $\left|c_{n}\right|$ be a monotone nonincreasing sequence, and let

$$
\sum_{n=1}^{\infty} \frac{\left|c_{n}\right|^{2}}{\sqrt{n}}<\infty, \quad \sum_{n=1}^{\infty} \frac{\omega_{n}}{n}<\infty, \quad \sup _{n \geq 0} \int_{0}^{1}|q(n+t)| d t<\infty .
$$

Then the absolutely continuous component of the spectrum of the operator $H_{d}$ fills the entire real line.

Theorem 1.6. Suppose

$$
\sum_{n=1}^{\infty} \frac{\left|c_{n}\right|}{\sqrt{n}}<\infty, \quad \sum_{n=1}^{\infty} \frac{\omega_{n}}{n}<\infty, \quad \sup _{n \geq 0} \int_{0}^{1}|q(n+t)| d t<\infty .
$$

Then the spectrum of the operator $H_{d}$ is purely absolutely continuous and fills the entire real line. Moreover, the spectral equation

$$
-\psi^{\prime \prime}-x \psi+q(x) \psi=E \psi, \quad E \in \mathbb{R}
$$

admits a solution $\psi_{+}$with the following asymptotic behavior as $x \rightarrow+\infty$ :

$$
\psi_{+}(x)=\frac{1}{\sqrt[4]{x}} \exp \left(i \int_{x_{0}}^{x}\left[\sqrt{t+E}-\frac{q(t)}{2 \sqrt{t+E}}\right] d t\right)(1+o(1)) .
$$

Here, $x_{0}$ satisfies $x_{0}+E>0$.

Remark. It should be noted that formula (1.5) makes it possible to describe the asymptotic behavior of an arbitrary solution of (1.4). 
We compare the statements of Theorems 1.1, 1.3, and 1.4 by the example of the following potential:

$$
q(x)=\sum_{n=1}^{\infty} \frac{n^{-\beta}}{\left|x-x_{n}\right|^{\alpha}} \omega(x-n) .
$$

Here, $x_{n}$ is an arbitrary real sequence such that $x_{n} \in[n+2 \epsilon, n+1-2 \epsilon]$ for $n \in \mathbb{N}$, $\epsilon \in\left[0, \frac{1}{4}\right], \beta>0, \alpha \in(0,1)$, and $\omega(x)$ is an arbitrary function of class $C^{\infty}(\mathbb{R})$ such that $\omega(x)=1$ for $x \in[\epsilon, 1-\epsilon]$ and $\omega(x)=0$ for $x \notin[0,1]$. For the Stark operator with potential of the form (1.6), Theorem 1.1 ensures the preservation of the absolutely continuous spectrum provided

$$
4 \beta>\max (1,2 \alpha) .
$$

Theorems 1.3 and 1.4 ensure the preservation of the absolutely continuous spectrum if the following weaker condition is satisfied:

$$
4 \beta>\max (1,2 \alpha)-1 .
$$

The applicability conditions for Theorems 1.3 and 1.4 turn out to be more free because the conditions on the potential $q$ are given in terms of Fourier-type integrals, which, besides the asymptotics, take into account the smoothness properties of the potential. Also, we note that, in the scale of the potentials satisfying an estimate of the form

$$
|q(x)| \leq \frac{C}{(1+|x|)^{\gamma}},
$$

Theorems 1.1, 1.3, and 1.4 are optimal and ensure the preservation of the absolutely continuous spectrum for $\gamma>\frac{1}{4}$ (see [8]).

It is more difficult to compare Theorems 1.2 and 1.4 and we restrict ourselves to some observations. We note that, on the one hand, condition (1.2) imposes quite strong restrictions on the local properties of the potential $q$ (it must be at least continuous), but, on the other hand, estimate (1.2) is an optimal condition on the behavior of the function $q$ at infinity [8]. As we saw above, Theorem 1.4 effectively shows how these properties affect the preservation of the absolutely continuous spectrum, without imposing any restrictions on the smoothness of the potential $q$.

We note that Theorems 1.3 and 1.4 give close results. I do not know whether one of the two theorems implies the other; however, for a certain class of potentials, Theorem 1.3 gives better results. It should also be mentioned that, in the present paper, the main object of study is a certain discrete system, and the results obtained for this system apply to other discrete problems of mathematical physics.

Finally, Theorem 1.6 is a by-product arising in the proof of Theorem 1.4 The conditions of Theorem 1.6 are also stated in terms of Fourier-type coefficients and are optimal in the scale of potentials satisfying (1.7). More precisely, Theorem 1.6 ensures the absence of a singular spectrum for $\gamma>\frac{1}{2}$. For the optimality of the estimate $\gamma>\frac{1}{2}$, see, e.g., the paper 2 .

We outline the proofs of Theorems 1.4 and 1.6. In the proofs, we use the relationship between the spectral properties of the operator $H_{d}$ and the asymptotic properties of the solutions of equation (1.4), obtained in the paper [10. To study the asymptotic properties of the solutions of (1.4), we reduce this equation to a discrete system of the form $\mathbf{a}(n+1)=T(n) \mathbf{a}(n)$ that describes how the solutions of equation (1.4) on the neighboring intervals $[n, n+1]$ and $[n+1, n+2]$ are related, where $n \in \mathbb{N}$. For the first time, the idea to reduce a differential equation of the form (1.4) to a discrete system was 
used in the paper [7] for a periodic potential of the form

$$
q(x)=V \sum_{n=-\infty}^{\infty} \delta(x-n) .
$$

In [7, the asymptotic behavior of the solutions of equation (1.4) with potential of the form (1.8) was described, and reasons were found why the spectral properties of the corresponding operator have been poorly understood.

Then, we describe the asymptotic behavior of the transition matrix $T(n)$ as $n \rightarrow \infty$, which is a relatively simple problem. To prove Theorem [1.6, it remains to perform a comparatively simple study of the above-mentioned system, which is presented at the end of the paper. The proof of Theorem 1.4 needs a subtler study of the discrete system. One of the difficulties here lies in the fact that the correction terms arising in the asymptotics of $T(n)$ can include a nontrivial dependence on the spectral parameter $E$. Therefore, the study is split into two stages. First, we treat a model system obtained, roughly speaking, from the given system by deleting the correction terms and, therefore, having an elementary dependence on $E$. To study this system, we use the method that was earlier suggested for localization of the absolutely continuous spectrum of the Schrödinger operator with slowly decaying potential [9]. This method is based on the spectral identities now called the BF-identities [1]. We note also that the same method was applied in [5] to systems with a simpler dependence on the spectrum parameter. Second, we compare the given system and the model system. Here, we use some wellknown ideas, which can be found in [1].

The structure of the present paper is as follows. In $\S 2$, we introduce the discrete system we need. In $\S 3$, we state the necessary results from [10] and discuss their relationship with the discrete system. $\S 4$ is devoted to the study of the model system and is divided into three subsections. In Subsection 1, we define the Weyl function. In Subsection 2, we obtain a spectral estimate of BF-type [1] for a model system of a specific form. In Subsection 3, we use passage to the limit to obtain a spectral estimate for the general model system. In $\S 5$, we prove two auxiliary statements to be applied later for comparing the given system and the model system. Finally, in $\S 6$, we prove Theorems 1.4, 1.6 and Corollary 1.5 .

\section{§2. Discrete System}

In this section, we transform equation (1.4) into a discrete system that describes the relationships among the solutions of equation (1.4) on the intervals $[n, n+1], n \in \mathbb{N}$. Usually, this procedure is employed when purely periodic equations are studied. The matrix connecting the solutions on the neighboring intervals is called the monodromy matrix. In contrast to the purely periodic case, the monodromy matrix for equation (1.4) depends on $n$. However, this dependence turns out to be very simple, which allows us to effectively study the discrete system obtained. We note that, for the equations of Wannier-Stark type, the procedure of transition from differential equations to discrete systems was used earlier in the papers [4, 7].

Remark. Instead of the intervals $[n, n+1]$, we could take an arbitrary sequence of intervals $\left[a_{n}, a_{n+1}\right]$ satisfying the conditions

$$
0<\inf _{n \geq 0}\left(a_{n+1}-a_{n}\right), \quad \sup _{n \geq 0}\left(a_{n+1}-a_{n}\right)<\infty .
$$

We have taken $a_{n}=n$ to simplify the calculation.

We introduce a specific basis of solutions of the spectral equation

$$
-\psi^{\prime \prime}-x \psi+q(x) \psi=E \psi, \quad E \in \mathbb{R} .
$$


Let $\theta_{n}(x)$ and $\varphi_{n}(x)$ be solutions of equation (2.1) such that

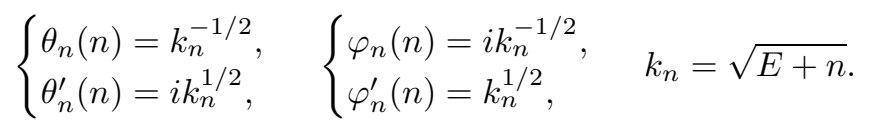

Here,

$$
W\left(\theta_{n}(x), \varphi_{n}(x)\right)=\theta_{n}(x) \varphi_{n}^{\prime}(x)-\theta_{n}^{\prime}(x) \varphi_{n}(x)=2 .
$$

We assume that $n>-E$, which implies that $k_{n}>0$.

On the interval $[n, n+1]$, an arbitrary solution $\psi$ of (2.1) can be represented in the form

$$
\begin{aligned}
\psi(x) & =\left(\mathbf{a}(n), \mathbf{w}_{n}(x)\right)_{r}, \quad \mathbf{a}(n)=\left(\begin{array}{c}
a_{1}(n) \\
a_{2}(n)
\end{array}\right), \\
\mathbf{w}_{n}(x) & =\left(\begin{array}{l}
\theta_{n}(x) \\
\varphi_{n}(x)
\end{array}\right), \quad x \in[n, n+1] .
\end{aligned}
$$

Here and below, the real inner product is marked by the index $r$,

$$
(\mathbf{a}, \mathbf{b})_{r}=a_{1} b_{1}+a_{2} b_{2} .
$$

Since the solution $\psi(x)$ must be continuously differentiable at the points $x=n$, the coefficients $a_{1}(n)$ and $a_{2}(n)$ must satisfy the following equation:

$$
\mathbf{a}(n+1)=T(n) \mathbf{a}(n),
$$

where the monodromy matrix $T(n)$ is given by

$$
T(n)=\left(\begin{array}{ll}
\theta_{n+1}(n+1) & \varphi_{n+1}(n+1) \\
\theta_{n+1}^{\prime}(n+1) & \varphi_{n+1}^{\prime}(n+1)
\end{array}\right)^{-1}\left(\begin{array}{cc}
\theta_{n}(n+1) & \varphi_{n}(n+1) \\
\theta_{n}^{\prime}(n+1) & \varphi_{n}^{\prime}(n+1)
\end{array}\right) .
$$

Relation (2.2) implies that $\operatorname{det} T(n)=1$.

Using the integral equations

$$
\begin{aligned}
& \theta_{n}(x)=\frac{1}{\sqrt{k_{n}}} e^{i k_{n}(x-n)}+\frac{1}{k_{n}} \int_{n}^{x} \sin \left\{k_{n}(x-t)\right\}(q(t)+n-t) \theta_{n}(t) d t, \\
& \varphi_{n}(x)=-\frac{1}{i \sqrt{k_{n}}} e^{-i k_{n}(x-n)}+\frac{1}{k_{n}} \int_{n}^{x} \sin \left\{k_{n}(x-t)\right\}(q(t)+n-t) \varphi_{n}(t) d t
\end{aligned}
$$

for $\theta_{n}$ and $\varphi_{n}$, we find the asymptotic behavior of the monodromy matrix as $n \rightarrow \infty$ :

$$
T(n)=e^{i \Delta(n) \sigma_{3}}\left(\begin{array}{cc}
1+O\left(d_{n}\right) & \frac{\bar{c}_{n}}{2 \sqrt{n}}+O\left(d_{n}\right) \\
\frac{c_{n}}{2 \sqrt{n}}+O\left(d_{n}\right) & 1+O\left(d_{n}\right)
\end{array}\right), \quad \sigma_{3}=\left(\begin{array}{cc}
1 & 0 \\
0 & -1
\end{array}\right) .
$$

Here,

$$
\begin{aligned}
\Delta(n) & =\frac{E}{2 \sqrt{n}}+\delta_{n}, \quad \delta_{n}=\sqrt{n}+\frac{1}{4 \sqrt{n}}\left(1-2 \int_{0}^{1} q(n+t) d t\right), \\
c_{n} & =\int_{0}^{1} q(n+t) e^{2 i \sqrt{n} t} d t, \quad d_{n}=\frac{\omega_{n}}{n}+n^{-3 / 2} \\
\omega_{n} & =\sup _{x \in[0,1]}\left|\int_{0}^{x} q(n+t) e^{2 i \sqrt{n} t} d t\right| .
\end{aligned}
$$

We plug

$$
\mathbf{a}(n)=e^{i \hat{\delta}_{n} \sigma_{3}} \mathbf{b}(n), \quad \hat{\delta}_{n}=\sum_{k=1}^{n-1} \delta_{k}
$$


in system (2.4). As a result, we obtain

$$
\mathbf{b}(n+1)=e^{i \frac{E}{2 \sqrt{n}} \sigma_{3}}\left(\begin{array}{cc}
1+O\left(d_{n}\right) & \frac{\bar{c}_{n}}{2 \sqrt{n}} e^{-2 i \hat{\delta}_{n}}+O\left(d_{n}\right) \\
\frac{c_{n}}{2 \sqrt{n}} e^{2 i \hat{\delta}_{n}}+O\left(d_{n}\right) & 1+O\left(d_{n}\right)
\end{array}\right) \mathbf{b}(n) .
$$

\section{§3. Subordinate solutions}

In the sequel, we need some results proved in [10. The required facts (see Theorems 3.2 and 3.3. can be stated as follows.

Definition 3.1. A solution $\psi^{u}$ of equation (1.4) is said to be subordinate if, for every solution $\psi$ such that $\psi^{u}$ and $\psi$ are linearly independent, we have

$$
\lim _{x \rightarrow+\infty}\left(\int_{0}^{x}\left|\psi^{u}(t)\right|^{2} d t / \int_{0}^{x}|\psi(t)|^{2} d t\right)=0 .
$$

Theorem 3.2 (Gilbert-Pearson). Assume that equation (1.4) admits no subordinate solutions for any $E \in \mathbb{R}$. Then the spectrum of the operator $H_{d}$ is absolutely continuous and fills the entire real line.

Theorem 3.3 (Gilbert-Pearson). Assume that, for almost all $E \in \mathbb{R}$ with respect to Lebesgue measure, equation (1.4) does not have subordinate solutions. Then the absolutely continuous spectrum of $H_{d}$ fills the entire real line.

Theorems 3.2 and 3.3 allow us to reduce the study of the absolutely continuous spectrum of $H_{d}$ to that of the asymptotic properties of the solutions of the discrete system (2.9).

Definition 3.4. A solution $\mathbf{b}^{u}$ of system (2.9) is said to be subordinate if for every solution $\mathbf{b}$ such that $\mathbf{b}^{u}$ and $\mathbf{b}$ are linearly independent, we have

$$
\lim _{N \rightarrow \infty}\left(\sum_{n=1}^{N} \frac{\left\|\mathbf{b}^{u}(n)\right\|^{2}}{\sqrt{n}} / \sum_{n=1}^{N} \frac{\|\mathbf{b}(n)\|^{2}}{\sqrt{n}}\right)=0 .
$$

Lemma 3.5. Assume that condition (1.1) is satisfied. Then equation (1.4) and system (2.9) have subordinate solutions simultaneously.

Proof. Using (2.3), (2.8), and the integral equations (2.5) and (2.6), we obtain

$$
\int_{n}^{n+1}|\psi(x)|^{2} d x=\frac{\|\mathbf{b}(n)\|^{2}}{\sqrt{n}}(1+o(1)) \quad \text { as } n \rightarrow \infty .
$$

This implies the claim.

Theorems 3.2 , 3.3, and Lemma 3.5 imply the following two statements.

Theorem 3.6. Suppose that condition (1.1) is satisfied and that, for all $E \in \mathbb{R}$, system (2.9) has no subordinate solutions. Then the spectrum of $H_{d}$ is absolutely continuous and fills the entire real line.

Theorem 3.7. Suppose that condition (1.1) is satisfied and that, for almost all $E \in \mathbb{R}$ with respect to Lebesgue measure, system (2.9) has no subordinate solutions. Then the absolutely continuous spectrum of $H_{d}$ fills the entire real line. 


\section{$\S 4$. Model Discrete SYSTEM}

In the study of system (2.9), certain difficulties arise because the correction terms $O\left(d_{n}\right)$ can depend on the spectral parameter $E$. Therefore, it is convenient to start with the following model system:

$$
\mathbf{s}_{n+1}=e^{i \frac{E}{2 \sqrt{n}} \sigma_{3}} W_{n} \mathbf{s}_{n}, \quad W_{n}=\left(\begin{array}{cc}
w_{n} & v_{n} \\
\bar{v}_{n} & w_{n}
\end{array}\right), \quad n \geq 1 .
$$

We assume that the matrices $W_{n}$ do not depend on $E$, the relation

$$
\operatorname{det} W_{n}=1
$$

is fulfilled, the $w_{n}$ are positive real parameters, and the $v_{n}$ are complex parameters. Observe that the matrix $W_{n}$ satisfies

$$
W_{n}=\sigma_{1} \bar{W}_{n} \sigma_{1}, \quad \sigma_{1}=\left(\begin{array}{cc}
0 & 1 \\
1 & 0
\end{array}\right) .
$$

4.1. The Weyl function. Let $\mathbf{g}(E)=\left\{\mathbf{g}_{n}(E)\right\}_{n=1}^{\infty}$ and $\mathbf{h}(E)=\left\{\mathbf{h}_{n}(E)\right\}_{n=1}^{\infty}$ be solutions of system (4.1) satisfying the initial conditions $\mathbf{g}_{1}(E)=\left(\begin{array}{l}1 \\ 0\end{array}\right)$ and $\mathbf{h}_{1}(E)=\left(\begin{array}{l}0 \\ 1\end{array}\right)$.

Theorem 4.1. There exists a Weyl function $m(E)$ that is regular for $\operatorname{Im}(E)>0$ and satisfies

$$
\mathbf{f}(E)=\mathbf{g}(E)+m(E) \mathbf{h}(E) \in l^{2}\left(\mathbb{N}, \mathbb{C}^{2}, n^{-1 / 2}\right) \quad \text { for all } E \text { with } \operatorname{Im}(E)>0 .
$$

Moreover, $|m(E)|<1$ for $\operatorname{Im}(E)>0$.

Remark. Let $l^{2}\left(\mathbb{N}, \mathbb{C}^{2}, n^{-1 / 2}\right)$ denote the Hilbert space of sequences of vectors $\mathbf{q}=$ $\left\{\mathbf{q}_{n}\right\}_{n=1}^{\infty}$ with the norm

$$
\|\mathbf{q}\|^{2}=\sum_{n=1}^{\infty} \frac{\left\|\mathbf{q}_{n}\right\|^{2}}{\sqrt{n}} .
$$

We do not dwell on the proof of Theorem 4.1. Instead, we note that it is quite standard and almost completely repeats the argument used in the proof of Theorem 7 in [5.

Corollary 4.2. For almost all $E \in \mathbb{R}$ with respect to Lebesgue measure, the Weyl function $m(E)$ has finite nontangential boundary values.

Proof. The inequality $|m(E)|<1$ for $\operatorname{Im}(E)>0$ shows that $m$ belongs to the Hardy class $H_{\infty}(\operatorname{Im}(E) \geq 0)$. This implies the claim; see [3].

Lemma 4.3. Let $\mathbf{f}(E)$ be the sequence of vector-valued functions introduced in Theorem 4.1. Then the following estimate is valid for $\operatorname{Im}(E)>0$ :

$$
\sum_{n=1}^{\infty} \frac{\left\|\mathbf{f}_{n}(E)\right\|^{2}}{\sqrt{n}} \leq \frac{3}{\operatorname{Im}(E)} e^{\operatorname{Im}(E)} .
$$

Proof. We put

$$
\left(\begin{array}{l}
f_{n}^{1}(E) \\
f_{n}^{2}(E)
\end{array}\right)=\mathbf{f}_{n}(E)
$$

A direct calculation shows that

$$
\left|f_{n}^{1}(E)\right|^{2}-\left|f_{n}^{2}(E)\right|^{2}=e^{\frac{\operatorname{Im}(E)}{\sqrt{n}}}\left|f_{n+1}^{1}(E)\right|^{2}-e^{-\frac{\operatorname{Im}(E)}{\sqrt{n}}}\left|f_{n+1}^{2}(E)\right|^{2} .
$$


Hence,

$$
\begin{aligned}
\left|f_{1}^{1}(E)\right|^{2}-\left|f_{n}^{1}(E)\right|^{2}+\left|f_{n}^{2}(E)\right|^{2}-\left|f_{1}^{2}(E)\right|^{2} \\
=\sum_{k=1}^{n-1}\left(\left|f_{k}^{1}(E)\right|^{2}-\left|f_{k+1}^{1}(E)\right|^{2}\right)-\sum_{k=1}^{n-1}\left(\left|f_{k}^{2}(E)\right|^{2}-\left|f_{k+1}^{2}(E)\right|^{2}\right) \\
=\sum_{k=1}^{n-1}\left(\left|f_{k}^{1}(E)\right|^{2}-\left|f_{k}^{2}(E)\right|^{2}\right)-\sum_{k=1}^{n-1}\left(\left|f_{k+1}^{1}(E)\right|^{2}-\left|f_{k+1}^{2}(E)\right|^{2}\right) \\
=\sum_{k=1}^{n-1}\left(e^{\frac{\operatorname{Im}(E)}{\sqrt{k}}}\left|f_{k+1}^{1}(E)\right|^{2}-e^{-\frac{\operatorname{Im}(E)}{\sqrt{k}}}\left|f_{k+1}^{2}(E)\right|^{2}\right)-\sum_{k=1}^{n-1}\left(\left|f_{k+1}^{1}(E)\right|^{2}-\left|f_{k+1}^{2}(E)\right|^{2}\right) \\
=\sum_{k=1}^{n-1}\left(e^{\frac{\operatorname{Im}(E)}{\sqrt{k}}}-1\right)\left|f_{k+1}^{1}(E)\right|^{2}+\sum_{k=1}^{n-1}\left(1-e^{-\frac{\operatorname{Im}(E)}{\sqrt{k}}}\right)\left|f_{k+1}^{2}(E)\right|^{2} .
\end{aligned}
$$

Passing to the limit as $n \rightarrow \infty$, we obtain

$$
\sum_{k=1}^{\infty}\left(e^{\frac{\operatorname{Im}(E)}{\sqrt{k}}}-1\right)\left|f_{k+1}^{1}(E)\right|^{2}+\sum_{k=1}^{\infty}\left(1-e^{-\frac{\operatorname{Im}(E)}{\sqrt{k}}}\right)\left|f_{k+1}^{2}(E)\right|^{2}=1-|m(E)|^{2} .
$$

It remains to use the elementary estimates

$$
\frac{\operatorname{Im}(E)}{\sqrt{k+1}} e^{-\operatorname{Im}(E)} \leq \frac{\operatorname{Im}(E)}{\sqrt{k}} e^{-\operatorname{Im}(E)} \leq 1-e^{-\frac{\operatorname{Im}(E)}{\sqrt{k}}} \leq e^{\frac{\operatorname{Im}(E)}{\sqrt{k}}}-1 .
$$

4.2. Spectral estimates. Let $W_{n}, n \in \mathbb{N}$, be a set of matrices. We consider the new set

$$
W_{n}^{l}= \begin{cases}W_{n} & \text { if } 1 \leq n \leq l-1, \quad I=\left(\begin{array}{ll}
1 & 0 \\
0 & 1
\end{array}\right), \\
I & \text { if } n \geq l,\end{cases}
$$

and the system (4.1) constructed by this set,

$$
\mathbf{s}_{n+1}=e^{i \frac{E}{2 \sqrt{n}} \sigma_{3}} W_{n}^{l} \mathbf{s}_{n}, \quad n \geq 1 .
$$

This system admits a solution $\mathbf{y}^{l}(E)$ satisfying

$$
\mathbf{y}_{n}^{l}(E)=e^{i \sum_{k=1}^{n-1} \frac{E}{2 \sqrt{k}}}\left(\begin{array}{l}
1 \\
0
\end{array}\right), \quad n \geq l .
$$

Let

$$
\left(\begin{array}{l}
a_{l}(E) \\
b_{l}(E)
\end{array}\right)=\mathbf{y}_{1}^{l}(E), \quad\left(\begin{array}{c}
\alpha_{n}^{l}(E) \\
\beta_{n}^{l}(E)
\end{array}\right)=\mathbf{y}_{n}^{l}(E), \quad n \geq 1 .
$$

It is easily seen that for the Weyl function $m_{l}(E)$ of system (4.5) we have

$$
m_{l}(E)=\frac{b_{l}(E)}{a_{l}(E)} .
$$

Lemma 4.4. The function $a_{l}(E)$ is regular for $E \in \mathbb{C}$ and does not vanish for $\operatorname{Im}(E) \geq 0$.

Proof. The function $a_{l}(E)$ is regular because the entries of the matrix $\left(e^{i \frac{E}{2 \sqrt{n}} \sigma_{3}} W_{n}^{l}\right)^{-1}$ are regular for $\operatorname{Im}(E) \geq 0$. Next, if $\operatorname{Im}(E) \geq 0$, then

$$
e^{\frac{\operatorname{Im}(E)}{\sqrt{n}}}\left|\alpha_{n+1}^{l}(E)\right|^{2}-e^{-\frac{\operatorname{Im}(E)}{\sqrt{n}}}\left|\beta_{n+1}^{l}(E)\right|^{2}=\left|\alpha_{n}^{l}(E)\right|^{2}-\left|\beta_{n}^{l}(E)\right|^{2} .
$$


Now, assume that there exists $E_{l}$ such that $a_{l}\left(E_{l}\right)=0$ and $\operatorname{Im}\left(E_{l}\right) \geq 0$. By (4.8), we have

$$
\begin{aligned}
-\left|b_{l}\left(E_{l}\right)\right|^{2} & =\left|\alpha_{1}^{l}\left(E_{l}\right)\right|^{2}-\left|\beta_{1}^{l}\left(E_{l}\right)\right|^{2}=e^{\operatorname{Im}\left(E_{l}\right)}\left|\alpha_{2}^{l}\left(E_{l}\right)\right|^{2}-e^{-\operatorname{Im}\left(E_{l}\right)}\left|\beta_{2}^{l}\left(E_{l}\right)\right|^{2} \\
& \geq\left|\alpha_{2}^{l}\left(E_{l}\right)\right|^{2}-\left|\beta_{2}^{l}\left(E_{l}\right)\right|^{2} \geq \cdots \geq\left|\alpha_{n}^{l}\left(E_{l}\right)\right|^{2}-\left|\beta_{n}^{l}\left(E_{l}\right)\right|^{2} \geq \cdots \\
& \geq\left|\alpha_{l}^{l}\left(E_{l}\right)\right|^{2}-\left|\beta_{l}^{l}\left(E_{l}\right)\right|^{2}=\left|\alpha_{l}^{l}\left(E_{l}\right)\right|^{2}>0 .
\end{aligned}
$$

The contradiction obtained completes the proof.

Lemma 4.5. For $E \in \mathbb{R}$, we have

$$
\left\{\begin{array}{l}
a_{l+1}=w_{l} a_{l}-\bar{v}_{l} e^{i \sum_{k=1}^{l-1} \frac{E}{\sqrt{k}} \bar{b}_{l},} \\
\bar{b}_{l+1}=w_{l} \bar{b}_{l}-v_{l} e^{-i \sum_{k=1}^{l-1} \frac{E}{\sqrt{k}}} a_{l} .
\end{array}\right.
$$

Proof. For all $E \in \mathbb{R}$ we can write

$$
\sigma_{1} e^{-i \frac{E}{2 \sqrt{n}} \sigma_{3}} \bar{W}_{n}^{l} \sigma_{1}=e^{i \frac{E}{2 \sqrt{n}} \sigma_{3}} W_{n}^{l}, \quad \sigma_{1}=\left(\begin{array}{ll}
0 & 1 \\
1 & 0
\end{array}\right) .
$$

It follows that $\sigma_{1} \overline{\mathbf{y}}^{l}$ is a solution of system (4.5) for $E \in \mathbb{R}$.

Using equation (4.6), we find the following values for the vector-valued functions $\sigma_{1} \overline{\mathbf{y}}_{n}^{l}$, $\mathbf{y}_{n}^{l}$, and $\mathbf{y}_{n}^{l+1}$ with $n=l$ :

$$
\mathbf{y}_{l}^{l}=e^{i \sum_{k=1}^{l-1} \frac{E}{2 \sqrt{k}}}\left(\begin{array}{l}
1 \\
0
\end{array}\right), \quad \sigma_{1} \overline{\mathbf{y}}_{l}^{l}=e^{-i \sum_{k=1}^{l-1} \frac{E}{2 \sqrt{k}}}\left(\begin{array}{l}
0 \\
1
\end{array}\right), \mathbf{y}_{l}^{l+1}=W_{l}^{-1} e^{i \sum_{k=1}^{l-1} \frac{E}{2 \sqrt{k}}}\left(\begin{array}{l}
1 \\
0
\end{array}\right) .
$$

Consequently,

$$
\mathbf{y}_{l}^{l+1}=w_{l} \mathbf{y}_{l}^{l}-e^{i \sum_{k=1}^{l-1} \frac{E}{\sqrt{k}}} \bar{v}_{l} \sigma_{1} \overline{\mathbf{y}}_{l}^{l} .
$$

The vector-valued functions $\sigma_{1} \overline{\mathbf{y}}_{n}^{l}, \mathbf{y}_{n}^{l}$, and $\mathbf{y}_{n}^{l+1}$ are solutions of the same system (4.5) for $1 \leq n \leq l$. Therefore, relation (4.9) can be extended to the values $1 \leq n \leq l$ :

$$
\mathbf{y}_{n}^{l+1}=w_{l} \mathbf{y}_{n}^{l}-e^{i \sum_{k=1}^{l-1} \frac{E}{\sqrt{k}}} \bar{v}_{l} \sigma_{1} \overline{\mathbf{y}}_{n}^{l} .
$$

Putting $n=1$ in (4.10), we obtain the required statement.

For $E \in \mathbb{R}$, we define

$$
d_{l}(E)=\frac{\bar{b}_{l}(E)}{a_{l}(E)} e^{i \sum_{k=1}^{l-1} \frac{E}{\sqrt{k}}} .
$$

Lemma 4.6. For $E \in \mathbb{R}$, we have the recurrence formula

$$
d_{l+1}(E)=e^{i \frac{E}{\sqrt{l}}} \frac{d_{l}(E)-u_{l}}{1-\bar{u}_{l} d_{l}(E)}, \quad u_{l}=\frac{v_{l}}{w_{l}} .
$$

Proof. This follows from Lemma 4.5 and identity (4.11).

Lemma 4.7. The function $d_{l}(E)$ extends up to a function regular in the domain $\operatorname{Im}(E) \geq 0$. Moreover, we have $\left|d_{l}(E)\right|<1$ for $\operatorname{Im}(E) \geq 0$.

Proof. We use induction. The base of induction is valid because $d_{1}(E) \equiv 0$. Now, suppose that the claim is valid for the function $d_{l}(E)$; we prove that it is valid for $d_{l+1}(E)$.

We define the function $d_{l+1}(E)$ for $\operatorname{Im}(E)>0$ by the recurrence formula (4.12). The relation $\operatorname{det} W_{l}=1$ implies that $\left|u_{l}\right|<1$, whence $\left|u_{l} d_{l}(E)\right|<1$. Now, the recurrence formula (4.12) shows that the function $d_{l+1}(E)$ is regular in the domain $\operatorname{Im}(E) \geq 0$.

It is easily seen that the linear fractional mapping

$$
w=\frac{z-u_{l}}{1-\bar{u}_{l} z}
$$


takes the unit disk $|z|<1$ to the unit disk $|w|<1$. Using this, the recurrence formula (4.12), and the fact that $\left|e^{i \frac{E}{\sqrt{l}}}\right| \leq 1$ for $\operatorname{Im}(E) \geq 0$, we see that $\left|d_{l+1}(E)\right|<1$ for $\operatorname{Im}(E) \geq 0$.

Lemma 4.8. Suppose $\left|u_{l}\right| \leq \frac{1}{16 \sqrt{l}} \leq \tau<1$,

$$
\sum_{l=1}^{\infty}\left|u_{l}\right| e^{-s_{l}}\left|\sum_{k=1}^{l-1} u_{k} e^{s_{k}}\right| \leq \varepsilon_{1}, \quad s_{l}=\sum_{k=1}^{l-1} \frac{1}{\sqrt{k}}
$$

and

$$
\sum_{l=1}^{\infty}\left|u_{l}\right| e^{-s_{l}} \sum_{k=1}^{l-1}\left|u_{k}\right|^{2} e^{s_{k}} \leq \varepsilon_{1}
$$

for some $\tau$ and $\varepsilon_{1}<\infty$. Then

$$
\sum_{l=1}^{\infty}\left|d_{l}(i)\right|\left|u_{l}\right| \leq \frac{4 \varepsilon_{1}}{1-\tau}<\infty
$$

Proof. By (4.12), we have

$$
d_{l+1}(i)=e^{-\frac{1}{\sqrt{l}}} \frac{d_{l}(i)-u_{l}}{1-\bar{u}_{l} d_{l}(i)}=e^{-\frac{1}{\sqrt{ }}}\left(d_{l}(i)-u_{l}\right)\left(1+\bar{u}_{l} d_{l}(i)+\frac{\bar{u}_{l}^{2} d_{l}(i)^{2}}{1-\bar{u}_{l} d_{l}(i)}\right) .
$$

Hence,

$$
e^{\frac{1}{\sqrt{l}}} d_{l+1}(i)=d_{l}(i)+\bar{u}_{l} d_{l}^{2}(i)-u_{l}+g_{l},
$$

where the function $g_{l}$ admits the estimate

$$
\left|g_{l}\right| \leq \frac{2\left|u_{l}\right|^{2}}{1-\tau}
$$

We substitute

$$
d_{l}(i)=e^{-s_{l}} e_{l}
$$

in (4.13), obtaining

$$
e_{l+1}=e_{l}+e^{-s_{l}} \bar{u}_{l} e_{l}^{2}+e^{s_{l}} \hat{g}_{l}, \quad e_{1}=0, \quad \hat{g}_{l}=g_{l}-u_{l} .
$$

Introducing the operator

$$
K e_{l}=\sum_{k=1}^{l-1} e^{-s_{k}} \bar{u}_{k} e_{k}^{2}
$$

we represent (4.14) in the form

$$
e_{l}=K e_{l}+r_{l}, \quad r_{l}=\sum_{k=1}^{l-1} e^{s_{k}} \hat{g}_{k} .
$$

We know a priori that equation (4.15) has a solution. To estimate the solutions of this equation, we consider the seminorms

$$
\left\|e_{l}\right\|_{1}=\sum_{l=1}^{\infty}\left|e _ { l } \left\|u_{l}\left|e^{-s_{l}}, \quad\left\|e_{l}\right\|_{2}=\sup _{l \geq 1}\right| e_{l}\left|\sum_{k=l}^{\infty}\right| u_{k} \mid e^{-s_{k}} .\right.\right.
$$


It is easily seen that

$$
\begin{aligned}
\left\|K e_{l}\right\|_{1} & \leq \sum_{l=1}^{\infty}\left|u_{l}\right| e^{-s_{l}} \sum_{k=1}^{l} e^{-s_{k}}\left|u_{k}\right|\left|e_{k}\right|^{2} \\
& =\sum_{k=1}^{\infty} e^{-s_{k}}\left|u_{k}\right|\left|e_{k}\right| \cdot\left|e_{k}\right| \sum_{l=k}^{\infty}\left|u_{l}\right| e^{-s_{l}} \leq\left\|e_{l}\right\|_{1}\left\|e_{l}\right\|_{2}, \\
& \left\|K e_{l}\right\|_{1} \leq\left\|e_{l}\right\|_{1}\left\|e_{l}\right\|_{2}, \quad\left\|r_{l}\right\|_{1} \leq \frac{3 \varepsilon_{1}}{1-\tau} .
\end{aligned}
$$

The a priori estimate

$$
\left|e_{l}\right|=\left|d_{l}(i)\right| e^{s_{l}} \leq e^{s_{l}}
$$

yields

$$
\left\|e_{l}\right\|_{2} \leq \sup _{l \geq 1} e^{s_{l}} \sum_{k=l}^{\infty}\left|u_{k}\right| e^{-s_{k}} \leq \sup _{l \geq 1} e^{s_{l}} \sum_{k=l}^{\infty} \frac{1}{16 \sqrt{k}} e^{-s_{k}} \leq \frac{1}{4} .
$$

Using (4.15), (4.16), and (4.17), we obtain

$$
\begin{aligned}
& \left\|e_{l}\right\|_{1}=\left\|K e_{l}+r_{l}\right\|_{1} \leq\left\|e_{l}\right\|_{1}\left\|e_{l}\right\|_{2}+\left\|r_{l}\right\|_{1} \\
& \left\|e_{l}\right\|_{1} \leq \frac{\left\|r_{l}\right\|_{1}}{1-\left\|e_{l}\right\|_{2}} \leq \frac{4 \varepsilon_{1}}{1-\tau}<\infty
\end{aligned}
$$

Hence,

$$
\sum_{l=1}^{\infty}\left|d_{l}(i)\right|\left|u_{l}\right|=\sum_{l=1}^{\infty}\left|e_{l}\right|\left|u_{l}\right| e^{-s_{l}}=\left\|e_{l}\right\|_{1} \leq \frac{4 \varepsilon_{1}}{1-\tau}<\infty .
$$

Lemma 4.9. Under the conditions of Lemma 4.8 , for every $\varepsilon \in\left[0, \frac{1}{2}\right]$, we have

$$
\int_{\mathbb{R}} \frac{\ln \left|a_{l}(E+i \varepsilon)\right|}{1+E^{2}} d E \leq \pi \sum_{n=1}^{l-1}\left|v_{n}\right|^{2}+\frac{10 \pi \varepsilon_{1}}{1-\tau} .
$$

Proof. By Lemma 4.5, we have

$$
a_{l+1}(E)=w_{l} a_{l}(E)-\bar{v}_{l} a_{l}(E) d_{l}(E)
$$

for all $E \in \mathbb{R}$. The functions $a_{l}(E), a_{l+1}(E)$, and $d_{l}(E)$ are regular in the $\operatorname{Im}(E) \geq 0$ and are continuous up to the boundary (see Lemmas 4.4 and 4.7). Now, the Privalov uniqueness theorem shows that relation (4.18) is valid also for $\operatorname{Im}(E) \geq 0$.

Taking the logarithm of both sides of (4.18) and using Lemmas 4.4, 4.7 and 4.8, we obtain

$$
\begin{aligned}
\ln a_{l+1}(E+i \varepsilon) & =\ln a_{l}(E+i \varepsilon)+\ln w_{l}+\ln \left(1-\frac{\bar{v}_{l}}{w_{l}} d_{l}(E+i \varepsilon)\right), \\
\int_{\mathbb{R}} \frac{\ln a_{l+1}(E+i \varepsilon)}{(1-\varepsilon)^{2}+E^{2}} d E & =\int_{\mathbb{R}} \frac{\ln a_{l}(E+i \varepsilon)}{(1-\varepsilon)^{2}+E^{2}} d E+\frac{\pi}{1-\varepsilon} \ln w_{l}+\frac{\pi}{1-\varepsilon} \ln \left(1-\frac{\bar{v}_{l}}{w_{l}} d_{l}(i)\right), \\
\int_{\mathbb{R}} \frac{\ln \left|a_{l+1}(E+i \varepsilon)\right|}{(1-\varepsilon)^{2}+E^{2}} d E & \leq \int_{\mathbb{R}} \frac{\ln \left|a_{l}(E+i \varepsilon)\right|}{(1-\varepsilon)^{2}+E^{2}} d E+2 \pi \ln w_{l}+2 \pi\left|d_{l}(i)\right|\left|v_{l}\right|, \\
\int_{\mathbb{R}} \frac{\ln \left|a_{l}(E+i \varepsilon)\right|}{1+E^{2}} d E & \leq \int_{\mathbb{R}} \frac{\ln \left|a_{l}(E+i \varepsilon)\right|}{(1-\varepsilon)^{2}+E^{2}} d E \leq 2 \pi \sum_{k=1}^{l-1} \ln w_{l}+\frac{10 \pi \varepsilon_{1}}{1-\tau} .
\end{aligned}
$$

To complete the proof of the lemma, it remains to use the estimate

$$
2 \ln w_{n} \leq\left|v_{n}\right|^{2},
$$

which follows from (4.2). 
Lemma 4.10. For $\operatorname{Im}(E) \geq 0$, we have

$$
\frac{1}{1-\left|m_{l}(E)\right|^{2}} \leq\left|a_{l}(E)\right|^{2} .
$$

Proof. By (4.8),

$$
\begin{aligned}
\left|a_{l}(E)\right|^{2}-\left|b_{l}(E)\right|^{2} & =e^{\operatorname{Im}(E)}\left|\alpha_{2}^{l}(E)\right|^{2}-e^{-\operatorname{Im}(E)}\left|\beta_{2}^{l}(E)\right|^{2} \\
& \geq e^{\operatorname{Im}(E)}\left(\left|\alpha_{2}^{l}(E)\right|^{2}-\left|\beta_{2}^{l}(E)\right|^{2}\right) \geq \cdots \\
& \geq e^{\sum_{n=1}^{l-1} \frac{\operatorname{Im}(E)}{\sqrt{n}}}\left(\left|\alpha_{l}^{l}(E)\right|^{2}-\left|\beta_{l}^{l}(E)\right|^{2}\right)=e^{\sum_{n=1}^{l-1} \frac{\operatorname{Im}(E)}{\sqrt{n}}}\left|\alpha_{l}^{l}(E)\right|^{2}=1 .
\end{aligned}
$$

Consequently,

$$
\frac{1}{\left|a_{l}(E)\right|^{2}} \leq 1-\frac{\left|b_{l}(E)\right|^{2}}{\left|a_{l}(E)\right|^{2}}=1-\left|m_{l}(E)\right|^{2} .
$$

Theorem 4.11. Under the conditions of Lemma 4.8, for every $\varepsilon \in\left[0, \frac{1}{2}\right]$, we have

$$
-\int_{\mathbb{R}} \frac{\ln \left(1-\left|m_{l}(E+i \varepsilon)\right|^{2}\right)}{1+E^{2}} d E \leq 2 \pi \sum_{n=1}^{l-1}\left|v_{n}\right|^{2}+\frac{20 \pi \varepsilon_{1}}{1-\tau} .
$$

This follows easily from Lemmas 4.9 and 4.10 .

\subsection{Passage to the limit.}

Lemma 4.12. Assume that the limit

$$
\lim _{n \rightarrow \infty} \sqrt{n}\left(W_{n}-I\right)=0
$$

exists and $E \in \mathbb{R}+i \varepsilon$, where $\varepsilon \in\left(0, \frac{1}{2}\right]$. Then

$$
\lim _{l \rightarrow \infty} m_{l}(E)=m(E)
$$

uniformly in $E$.

Proof. Consider the operator

$$
M \mathbf{q}_{n}=\mathbf{q}_{n+1}-e^{i \frac{E}{2 \sqrt{n}} \sigma_{3}} W_{n} \mathbf{q}_{n}, \quad \operatorname{Im}(E)>0, \quad n \in \mathbb{N},
$$

with the domain

$$
\operatorname{Dom}(M)=\left\{\mathbf{q}: \mathbf{q}_{1}=\left(\begin{array}{l}
0 \\
.
\end{array}\right)\right\}
$$

The kernel of $M$ is trivial. The inverse operator $M^{-1}$ is well defined on $l^{2}\left(\mathbb{N}, \mathbb{C}^{2}, \sqrt{n}\right)$ by the formula

$$
M^{-1} \mathbf{q}_{n}=\sum_{k=1}^{\infty} G(n, k) \mathbf{q}_{k}, \quad n \in \mathbb{N}
$$

where

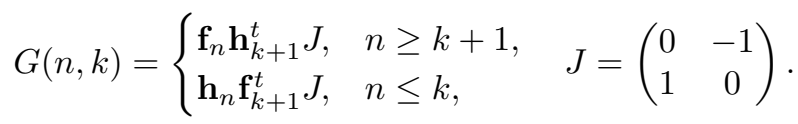

Here ${ }^{t}$ stands for transposition, and $\mathbf{h}, \mathbf{f}$ are the sequences of vector-valued functions as in Theorem 4.1 .

Let $M_{l}$ denote the operator $M$ constructed for the set (4.4). On $l^{2}\left(\mathbb{N}, \mathbb{C}^{2}, \sqrt{n}\right)$, we have the resolvent identity

$$
M^{-1}-M_{l}^{-1}=M_{l}^{-1}\left(M_{l}-M\right) M^{-1} .
$$


We consider the following element of $l^{2}\left(\mathbb{N}, \mathbb{C}^{2}, \sqrt{n}\right)$ :

$$
\mathbf{q}=\left\{\mathbf{q}_{n}\right\}_{n=1}^{\infty}, \quad \mathbf{q}_{n}=\left\{\begin{array}{lll}
\mathbf{g}_{2} & \text { if } & n=1 \\
0 & \text { if } & n \geq 2
\end{array}\right.
$$

For $l \geq 2$, we have

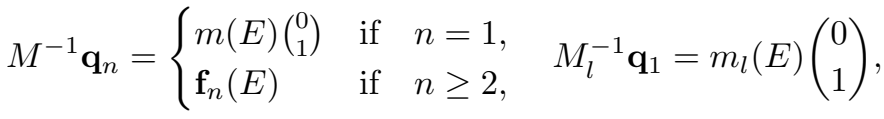

$$
\begin{aligned}
& M_{l}^{-1}\left(M_{l}-M\right) M^{-1} \mathbf{q}_{1}=\left(\begin{array}{l}
0 \\
1
\end{array}\right) \sum_{n \geq l}\left(\mathbf{f}_{n+1}^{l}(E)\right)^{t} e^{i \frac{E}{2 \sqrt{n}} \sigma_{3}}\left(W_{n}-I\right) \mathbf{f}_{n}(E) .
\end{aligned}
$$

Now, we apply both sides of (4.22) to the element $\mathbf{q}$, obtaining

$$
m(E)-m_{l}(E)=\sum_{n \geq l}\left(\mathbf{f}_{n+1}^{l}(E)\right)^{t} e^{i \frac{E}{2 \sqrt{n}} \sigma_{3}}\left(W_{n}-I\right) \mathbf{f}_{n}(E),
$$

whence

$$
\left|m(E)-m_{l}(E)\right| \leq e^{\varepsilon} \sup _{n \geq l}\left(\sqrt{n}\left\|W_{n}-I\right\|\right) \sum_{n=1}^{\infty} \frac{\left\|\mathbf{f}_{n}^{l}(E)\right\|^{2}}{\sqrt{n}} \sum_{n=1}^{\infty} \frac{\left\|\mathbf{f}_{n}(E)\right\|^{2}}{\sqrt{n}} .
$$

It remains to use Lemma 4.3 .

Theorem 4.13. Under the conditions of Lemma 4.8, suppose that the limit

$$
\lim _{n \rightarrow \infty} \sqrt{n}\left(W_{n}-I\right)=0
$$

exists and

$$
\sum_{n=1}^{\infty}\left|v_{n}\right|^{2}<\infty
$$

Then

$$
\tau=\sup _{n \geq 1}\left|v_{n}\right| / w_{n}<1
$$

and we have

$$
-\int_{\mathbb{R}} \frac{\ln \left(1-|m(E)|^{2}\right)}{1+E^{2}} d E \leq 2 \pi \sum_{n=1}^{\infty}\left|v_{n}\right|^{2}+\frac{20 \pi \varepsilon_{1}}{1-\tau}<\infty .
$$

Proof. Since the series $\sum_{n \geq 1}\left|v_{n}\right|^{2}$ converges, we have $\lim _{n \rightarrow \infty}\left|v_{n}\right|=0$ and, as a consequence, $\sup _{n \geq 1} w_{n}<\infty$. Recalling (4.2), we obtain

$$
\tau^{2}=\sup _{n \geq 1}\left|v_{n}\right|^{2} / w_{n}^{2}=1-\inf _{n \geq 1} w_{n}^{-2}<1 .
$$

By Lemma 4.12 for every $\varepsilon \in\left(0, \frac{1}{2}\right]$, we can pass to the limit as $l \rightarrow \infty$ under the integral sign in (4.19). Therefore, by Theorem 4.11,

$$
-\int_{\mathbb{R}} \frac{\ln \left(1-|m(E+i \varepsilon)|^{2}\right)}{1+E^{2}} d E \leq 2 \pi \sum_{n=1}^{\infty}\left|v_{n}\right|^{2}+\frac{20 \pi \varepsilon_{1}}{1-\tau}<\infty .
$$

Corollary 4.2 shows that, for almost all $E \in \mathbb{R}$ with respect to Lebesgue measure, the Weyl function $m(E+i \varepsilon)$ has a finite limit as $\varepsilon \rightarrow 0+0$. Now, applying Fatou's lemma on convergence as $\varepsilon \rightarrow 0+0$, we obtain the required estimate. 


\section{§5. Auxiliary Results}

To compare the behavior of solutions of systems (2.8) and (4.1) for large values of the argument, we need two auxiliary lemmas, which are stated below. The basic ideas of their proofs are taken from [11.

Lemma 5.1. Let $F_{n}$ be a matrix solution of the system

$$
F_{n+1}=e^{i \frac{E}{2 \sqrt{n}} \sigma_{3}} W_{n} F_{n}, \quad F_{1}=I .
$$

Assume that the conditions of Lemma 4.8, estimate (4.23), and the conditions

$$
\begin{aligned}
& \sup _{n \geq 1} \sqrt{n}\left\|W_{n}-I\right\| \leq \frac{1}{4}, \\
& \lim _{n \rightarrow \infty} \sqrt{n}\left(W_{n}-I\right)=0
\end{aligned}
$$

are satisfied. Let $a_{n}$ be a sequence of nonnegative numbers such that the series $\sum_{n \geq 1} a_{n}$ converges. Then, for almost all $E \in \mathbb{R}$ with respect to Lebesgue measure, there exist constants $C_{1}(E)$ and $C_{2}(E)$ such that

$$
\begin{array}{r}
\sum_{n=1}^{\infty} a_{n}\left\|F_{n}\right\|\left\|F_{n+1}\right\| \leq C_{1}(E)<\infty, \\
\liminf _{N \rightarrow \infty} \frac{1}{\sqrt{N}} \sum_{n=1}^{N} \frac{\left\|F_{n}\right\|^{2}}{\sqrt{n}} \leq C_{2}(E)<\infty .
\end{array}
$$

Proof. Using the resolvent identity (4.22), we obtain

$$
\left(I-M_{1}^{-1} \hat{E}(\hat{W}-I)\right) M^{-1}=M_{1}^{-1}
$$

where $\hat{E}$ and $\hat{W}$ are the operators of multiplication by $e^{i \frac{E}{2 \sqrt{n}} \sigma_{3}}$ and $W_{n}$, respectively.

Let $G_{1}(n, k)$ be the Green function of the operator $M_{1}^{-1}$ (see (4.21)). For $\operatorname{Im}(E)>0$, simple calculations yield the formulas

$$
\begin{aligned}
& G_{1}(n, k)=\exp \left(i \sum_{m=k+1}^{n-1} \frac{E}{2 \sqrt{m}}\right)\left(\begin{array}{cc}
1 & 0 \\
0 & 0
\end{array}\right) \quad \text { for } n \geq k+1, \\
& G_{1}(n, k)=\exp \left(i \sum_{m=n}^{k} \frac{E}{2 \sqrt{m}}\right)\left(\begin{array}{cc}
0 & 0 \\
0 & -1
\end{array}\right) \quad \text { for } n \leq k .
\end{aligned}
$$

Hence,

$$
\begin{gathered}
\left\|G_{1}(n, k)\right\| \leq e^{-\operatorname{Im}(E)|\sqrt{n}-\sqrt{k+1}|} \\
\left\|G_{1}(n, k) e^{i \frac{E}{2 \sqrt{k}} \sigma_{3}}\right\| \leq e^{-\operatorname{Im}(E)|\sqrt{n}-\sqrt{k}|} \text { for } \operatorname{Im}(E)>0, \\
\sup _{n \geq 1} \sum_{m=1}^{\infty} \frac{\left\|G_{1}(n, m)\right\|}{\sqrt{m}} \leq 3, \quad \sup _{k \geq 1} \sum_{n=1}^{\infty} \frac{\left\|G_{1}(n, k)\right\|}{\sqrt{n}} \leq 3 \quad \text { for } \operatorname{Im}(E) \geq 2 .
\end{gathered}
$$


Now, we consider $M_{1}^{-1}$ as an operator from $l^{2}\left(\mathbb{N}, \mathbb{C}^{2}, \sqrt{n}\right)$ to $l^{2}\left(\mathbb{N}, \mathbb{C}^{2}, n^{-1 / 2}\right)$. For $\operatorname{Im}(E) \geq 2$, we have

$$
\begin{aligned}
& \left\|M_{1}^{-1} ; l^{2}\left(\mathbb{N}, \mathbb{C}^{2}, \sqrt{n}\right) \rightarrow l^{2}\left(\mathbb{N}, \mathbb{C}^{2}, n^{-1 / 2}\right)\right\|^{2} \\
& \quad=\sup _{\left\|\mathbf{q} ; l^{2}\left(\mathbb{N}, \mathbb{C}^{2}, \sqrt{n}\right)\right\|=1}\left\|M_{1}^{-1} \mathbf{q} ; l^{2}\left(\mathbb{N}, \mathbb{C}^{2}, n^{-1 / 2}\right)\right\|^{2} \\
& \quad=\sup _{\left\|\mathbf{q} ; l^{2}\left(\mathbb{N}, \mathbb{C}^{2}, \sqrt{n}\right)\right\|=1} \sum_{n=1}^{\infty} \frac{1}{\sqrt{n}}\left\|\sum_{k=1}^{\infty} G_{1}(n, k) \mathbf{q}_{k}\right\|^{2} \\
& \quad \leq \sup _{\left\|\mathbf{q} ; l^{2}\left(\mathbb{N}, \mathbb{C}^{2}, \sqrt{n}\right)\right\|=1} \sum_{n=1}^{\infty} \frac{1}{\sqrt{n}} \sum_{m=1}^{\infty} \frac{\left\|G_{1}(n, m)\right\|}{\sqrt{m}} \sum_{k=1}^{\infty} \sqrt{k}\left\|G_{1}(n, k)\right\|\left\|\mathbf{q}_{k}\right\|^{2} \\
& \quad \leq \sup _{n \geq 1} \sum_{m=1}^{\infty} \frac{\left\|G_{1}(n, m)\right\|}{\sqrt{m}} \sup _{k \geq 1} \sum_{n=1}^{\infty} \frac{\left\|G_{1}(n, k)\right\|}{\sqrt{n}} \sup _{\left\|\mathbf{q} ; l^{2}\left(\mathbb{N}, \mathbb{C}^{2}, \sqrt{k}\right)\right\|=1} \sum_{k=1}^{\infty} \sqrt{k}\left\|\mathbf{q}_{k}\right\|^{2} \leq 9 .
\end{aligned}
$$

Similarly,

$$
\left\|M_{1}^{-1} \hat{E} ; l^{2}\left(\mathbb{N}, \mathbb{C}^{2}, \sqrt{n}\right) \rightarrow l^{2}\left(\mathbb{N}, \mathbb{C}^{2}, n^{-1 / 2}\right)\right\| \leq 3 \quad \text { for } \operatorname{Im}(E) \geq 2 .
$$

Using condition (5.2), we can easily check that

$$
\left\|\hat{W}-I ; l^{2}\left(\mathbb{N}, \mathbb{C}^{2}, n^{-1 / 2}\right) \rightarrow l^{2}\left(\mathbb{N}, \mathbb{C}^{2}, \sqrt{n}\right)\right\| \leq \frac{1}{4} .
$$

Therefore, by (5.7),

$$
\left\|M_{1}^{-1} \hat{E}(\hat{W}-I) ; l^{2}\left(\mathbb{N}, \mathbb{C}^{2}, n^{-1 / 2}\right) \rightarrow l^{2}\left(\mathbb{N}, \mathbb{C}^{2}, n^{-1 / 2}\right)\right\| \leq \frac{3}{4} \quad \text { for } \operatorname{Im}(E) \geq 2 .
$$

Estimate (5.8) ensures that the operator $I-M_{1}^{-1} \hat{E}(\hat{W}-I)$ is invertible for $\operatorname{Im}(E) \geq 2$. Now, we use (5.4) to estimate the norm of the operator $M^{-1}$ :

$$
\begin{aligned}
&\left\|M^{-1} ; l^{2}\left(\mathbb{N}, \mathbb{C}^{2}, \sqrt{n}\right) \rightarrow l^{2}\left(\mathbb{N}, \mathbb{C}^{2}, n^{-1 / 2}\right)\right\| \\
& \leq \|(I-\left.M_{1}^{-1} \hat{E}(\hat{W}-I)\right)^{-1} ; l^{2}\left(\mathbb{N}, \mathbb{C}^{2}, n^{-1 / 2}\right) \rightarrow l^{2}\left(\mathbb{N}, \mathbb{C}^{2}, n^{-1 / 2}\right) \| \\
& \quad \times\left\|M_{1}^{-1} ; l^{2}\left(\mathbb{N}, \mathbb{C}^{2}, \sqrt{n}\right) \rightarrow l^{2}\left(\mathbb{N}, \mathbb{C}^{2}, n^{-1 / 2}\right)\right\| \leq 12 \quad \text { for } \operatorname{Im}(E) \geq 2 .
\end{aligned}
$$

To estimate the norm of the matrix $G(l, l-1)$, we use the iterated resolvent identity

$$
M^{-1}=M_{1}^{-1}+M_{1}^{-1}\left(M_{1}-M\right) M_{1}^{-1}+M_{1}^{-1}\left(M_{1}-M\right) M^{-1}\left(M_{1}-M\right) M_{1}^{-1} .
$$

We have

$$
\begin{aligned}
G(l, l-1)=G_{1}(l, l-1) & +\sum_{k=1}^{\infty} G_{1}(l, k)\left(W_{k}-I\right) G_{1}(k, l-1) \\
& +\sum_{k=1}^{\infty} \sum_{n=1}^{\infty} G_{1}(l, k)\left(W_{k}-I\right) G(k, n)\left(W_{n}-I\right) G_{1}(n, l-1) .
\end{aligned}
$$


By estimates (5.5) and (5.6), we obtain

$$
\begin{aligned}
\|G(l, l-1)\| \leq & +\sum_{k=1}^{\infty} \frac{1}{4 \sqrt{k}}\left\|G_{1}(l, k)\right\|\left\|G_{1}(k, l-1)\right\| \\
& +\sum_{k=1}^{\infty} \frac{1}{4 \sqrt{k}}\left\|G_{1}(l, k)\right\|\left\|\sum_{n=1}^{\infty} G(k, n)\left(W_{n}-I\right) G_{1}(n, l-1)\right\| \\
\leq & \frac{7}{4}+\frac{1}{4}\left(\sum_{k=1}^{\infty} \frac{1}{\sqrt{k}}\left\|G_{1}(l, k)\right\|^{2}\right)^{1 / 2} \\
& \times\left(\sup _{\left\|\mathbf{q} ; l^{2}\left(\mathbb{N}, \mathbb{C}^{2}, \sqrt{n}\right)\right\| \leq 1} \sum_{k=1}^{\infty} \frac{1}{\sqrt{k}}\left\|\sum_{n=1}^{\infty} G(k, n) \mathbf{q}_{n}\right\|^{2}\right)^{1 / 2} \\
\leq & 2+\frac{1}{2}\left\|M^{-1} ; l^{2}\left(\mathbb{N}, \mathbb{C}^{2}, \sqrt{n}\right) \rightarrow l^{2}\left(\mathbb{N}, \mathbb{C}^{2}, n^{-1 / 2}\right)\right\| \leq 8 .
\end{aligned}
$$

Finally, for $l \geq 2$ and $\operatorname{Im}(E) \geq 2$, we arrive at the estimate

$$
\|G(l, l-1)\| \leq 8 .
$$

Theorem 4.1] shows that the vector-valued functions $\mathbf{f}_{n}$ and $\mathbf{h}_{n}$ are regular and bounded in the domain $\operatorname{Im}(E)>0$ for any fixed $n \geq 1$. From the representation (4.21) for the matrix $G(l, l-1, E)$, it follows that this matrix is regular and bounded in the same domain. Now, applying the residue theorem, we can easily evaluate the following integral:

$$
\int_{\mathbb{R}} \frac{G(l, l-1, E+i \varepsilon)}{4+E^{2}} d E=\frac{\pi}{2} G(l, l-1,2 i), \quad \varepsilon>0 .
$$

Since the matrix $G(l, l-1, E)$ is uniformly bounded for $\operatorname{Im}(E)>0$, we can apply the Lebesgue dominated convergence theorem and Corollary 4.2 to pass to the limit as $\varepsilon \rightarrow$ $0+0$ under the integral sign in (5.10). Therefore, by (5.9),

$$
\left\|\int_{\mathbb{R}} \frac{G(l, l-1, E)}{4+E^{2}} d E\right\| \leq 4 \pi .
$$

Relation (4.3) shows that, for $E \in \mathbb{R}$, the solutions $\mathbf{h}$ and $\mathbf{g}$ of system (4.1) satisfy $\mathbf{g}=\sigma_{1} \overline{\mathbf{h}}$. We denote the components of the vector $\mathbf{h}_{l}$ by $h_{l}^{1}$ and $h_{l}^{2}$. Using (4.21), we obtain

$$
\begin{aligned}
G(l, l-1, E) & =\left(\sigma_{1} \overline{\mathbf{h}}_{l}+m(E) \mathbf{h}_{l}\right) \mathbf{h}_{l}^{t} J \\
& =\left(\left(\begin{array}{cc}
h_{l}^{1} \overline{h_{l}^{2}} & \left|h_{l}^{2}\right|^{2} \\
\left|h_{l}^{1}\right|^{2} & h_{l}^{2} \overline{h_{l}^{1}}
\end{array}\right)+m(E)\left(\begin{array}{cc}
\left(h_{l}^{1}\right)^{2} & h_{l}^{1} h_{l}^{2} \\
h_{l}^{1} h_{l}^{2} & \left(h_{l}^{2}\right)^{2}
\end{array}\right)\right)\left(\begin{array}{cc}
0 & -1 \\
1 & 0
\end{array}\right) \\
& =\left(\begin{array}{cc}
\left|h_{l}^{2}\right|^{2}+m(E) h_{l}^{1} h_{l}^{2} & -h_{l}^{1} \overline{h_{l}^{2}}-m(E)\left(h_{l}^{1}\right)^{2} \\
h_{l}^{2} \overline{h_{l}^{1}}+m(E)\left(h_{l}^{2}\right)^{2} & -\left|h_{l}^{1}\right|^{2}-m(E) h_{l}^{1} h_{l}^{2}
\end{array}\right) .
\end{aligned}
$$

Subtracting the diagonal entries in (5.12) and using (5.11), we see that

$$
\int_{\mathbb{R}} \frac{1-|m(E)|}{4+E^{2}}\left\|\mathbf{h}_{l}\right\|^{2} d E \leq\left|\int_{\mathbb{R}} \frac{\left|h_{l}^{1}\right|^{2}+\left|h_{l}^{2}\right|^{2}+2 m(E) h_{l}^{1} h_{l}^{2}}{4+E^{2}} d E\right| \leq 8 \pi .
$$

For $E \in \mathbb{R}$ we have $F_{l}=\left(\sigma_{1} \overline{\mathbf{h}}_{l}, \mathbf{h}_{l}\right)$. Thus, (5.13) yields the estimate

$$
\int_{\mathbb{R}} \frac{1-|m(E)|}{4+E^{2}}\left\|F_{l}\right\|^{2} d E \leq 16 \pi
$$


which, in its turn, implies that

$$
\int_{\mathbb{R}} \frac{1-|m(E)|}{4+E^{2}} \sum_{n=1}^{N} a_{n}\left\|F_{n}\right\|\left\|F_{n+1}\right\| d E \leq 16 \pi \sum_{n=1}^{\infty} a_{n} .
$$

Now, we apply the Beppo Levi theorem to inequality (5.15), observing that $|m(E)|<1$ almost everywhere (see Theorem 4.13). As a result, we conclude that the series $\sum_{n=1}^{\infty} a_{n}\left\|F_{n}\right\|\left\|F_{n+1}\right\|$ converges for almost all $E \in \mathbb{R}$ with respect to Lebesgue measure.

The second estimate in the lemma follows from the inequalities

$$
\begin{aligned}
\int_{\mathbb{R}} \frac{1-|m(E)|}{4+E^{2}} \liminf _{N \rightarrow \infty} \frac{1}{\sqrt{N}} \sum_{n=1}^{N} \frac{\left\|F_{n}\right\|^{2}}{\sqrt{n}} d E \\
\quad \leq \liminf _{N \rightarrow \infty} \frac{1}{\sqrt{N}} \sum_{n=1}^{N} \frac{1}{\sqrt{n}} \int_{\mathbb{R}} \frac{1-|m(E)|}{4+E^{2}}\left\|F_{n}\right\|^{2} d E \leq 32 \pi
\end{aligned}
$$

and the estimate $|m(E)|<1$, which is valid for almost all $E \in \mathbb{R}$ with respect to Lebesgue measure (see Theorem 4.13).

Lemma 5.2. Let $B_{n}$ be a matrix solution of the system

$$
B_{n+1}=K_{n} B_{n}, \quad B_{1}=I,
$$

with matrix $K_{n}$ satisfying $\operatorname{det} K_{n}=1$. If

$$
\liminf _{N \rightarrow \infty} \frac{1}{\sqrt{N}} \sum_{n=1}^{N} \frac{\left\|B_{n}\right\|^{2}}{\sqrt{n}}<\infty,
$$

then system (5.16) has no subordinate solutions.

Proof. Let $\mathbf{u}$ and $\mathbf{v}$ be two linearly independent solutions of system (5.16). Multiplying one of them by an appropriate constant, we may assume that $\operatorname{det}\left(\mathbf{u}_{1}, \mathbf{v}_{1}\right)=1$. Since $\operatorname{det} K_{n}=1$, we have

$$
\operatorname{det}\left(\mathbf{u}_{n}, \mathbf{v}_{n}\right)=\operatorname{det}\left(\mathbf{u}_{1}, \mathbf{v}_{1}\right)=1
$$

whence

$$
\frac{1}{\sqrt{n}} \leq \frac{\left\|\mathbf{u}_{n}\right\|\left\|\mathbf{v}_{n}\right\|}{\sqrt{n}}
$$

Summing from 1 to $N$, we obtain

$$
\frac{N}{4} \leq\left(\sum_{n=1}^{N} \frac{1}{\sqrt{n}}\right)^{2} \leq\left(\sum_{n=1}^{N} \frac{\left\|\mathbf{u}_{n}\right\|\left\|\mathbf{v}_{n}\right\|}{\sqrt{n}}\right)^{2} \leq \sum_{n=1}^{N} \frac{\left\|\mathbf{u}_{n}\right\|^{2}}{\sqrt{n}} \sum_{n=1}^{N} \frac{\left\|\mathbf{v}_{n}\right\|^{2}}{\sqrt{n}} .
$$

Since $u_{n}=B_{n} u_{1}$, we have

$$
\frac{\sum_{n=1}^{N} \frac{\left\|\mathbf{u}_{n}\right\|^{2}}{\sqrt{n}}}{\sum_{n=1}^{N} \frac{\left\|\mathbf{v}_{n}\right\|^{2}}{\sqrt{n}}} \leq \frac{4}{N}\left(\sum_{n=1}^{N} \frac{\left\|\mathbf{u}_{n}\right\|^{2}}{\sqrt{n}}\right)^{2} \leq 4\left\|\mathbf{u}_{1}\right\|^{4}\left(\frac{1}{\sqrt{N}} \sum_{n=1}^{N} \frac{\left\|B_{n}\right\|^{2}}{\sqrt{n}}\right)^{2} .
$$

Now, assume that $\mathbf{v}$ is a subordinate solution. Then, by (5.17) and (5.18), there exists a sequence $N_{j}$ such that

$$
\sum_{n=1}^{N_{j}} \frac{\left\|\mathbf{u}_{n}\right\|^{2}}{\sqrt{n}} / \sum_{n=1}^{N_{j}} \frac{\left\|\mathbf{v}_{n}\right\|^{2}}{\sqrt{n}} \leq C_{3}<\infty, \quad j=1,2, \ldots,
$$

which contradicts the definition of a subordinate solution. 


\section{§6. Proof of the Main Result}

Proof of Theorem 1.4. We represent system (2.9) in the form

$$
B_{n+1}=e^{i \frac{E}{2 \sqrt{n}} \sigma_{3}}\left(W_{n}+V_{n}(E)\right) B_{n}, \quad B_{1}=I, \quad E \in \mathbb{R} .
$$

Here,

$$
\begin{aligned}
& W_{n}=I \text { for } 1 \leq n \leq N_{1}-1, \\
& W_{n}=\left(\begin{array}{ll}
\sqrt{1+\frac{\left|c_{n}\right|^{2}}{4 n}} & \frac{\bar{c}_{n}}{2 \sqrt{n}} e^{-2 i \hat{\delta}_{n}} \\
\frac{c_{n}}{2 \sqrt{n}} e^{2 i \hat{\delta}_{n}} & \sqrt{1+\frac{\left|c_{n}\right|^{2}}{4 n}}
\end{array}\right) \quad \text { for } n \geq N_{1},
\end{aligned}
$$

and the matrix $V_{n}(E)$ admits the estimate $\left\|V_{n}(E)\right\|=O\left(d_{n}\right)$, where, in general, $O\left(d_{n}\right)$ depends on $E$. The assumptions of Theorem 1.4 imply that $\lim _{n \rightarrow \infty} c_{n}=0$. Therefore, there exists a constant $N_{1} \geq 1$ such that $\left|c_{n}\right| \leq \frac{1}{4}$, and the assumptions of Lemma 4.8 are fulfilled.

We consider the auxiliary system

$$
F_{n+1}=e^{i \frac{E}{2 \sqrt{n}} \sigma_{3}} W_{n} F_{n}, \quad F_{1}=I, \quad E \in \mathbb{R} .
$$

It is easily seen that the matrix $W_{n}$ satisfies all the assumptions of Lemma 5.1, and the assumptions of Theorem 1.4 imply the convergence of the series $\sum_{n=1}^{\infty}\left\|V_{n}\right\|$. It follows that the solution $F_{n}$ satisfies estimates (5.3) for $a_{n}=V_{n}$. The change $B_{n}=F_{n} U_{n}$ transforms system (6.1) to

$$
U_{n+1}=\left(I+F_{n+1}^{-1} e^{i \frac{E}{2 \sqrt{n}} \sigma_{3}} V_{n} F_{n}\right) U_{n}, \quad U_{1}=I, \quad E \in \mathbb{R} .
$$

Hence,

$$
\left\|U_{n}\right\| \leq \prod_{n=1}^{N-1}\left(1+\left\|V_{n}\right\|\left\|F_{n}\right\|\left\|F_{n+1}\right\|\right) \leq \exp \left(\sum_{n=1}^{\infty}\left\|V_{n}\right\|\left\|F_{n}\right\|\left\|F_{n+1}\right\|\right) \leq e^{C_{1}(E)}<\infty
$$

for almost all $E \in \mathbb{R}$ with respect to Lebesgue measure.

Using the second estimate in (5.3), we obtain

$$
\liminf _{N \rightarrow \infty} \frac{1}{\sqrt{N}} \sum_{n=1}^{N} \frac{\left\|B_{n}\right\|^{2}}{\sqrt{n}} \leq e^{2 C_{1}(E)} \liminf _{N \rightarrow \infty} \frac{1}{\sqrt{N}} \sum_{n=1}^{N} \frac{\left\|F_{n}\right\|^{2}}{\sqrt{n}} \leq e^{2 C_{1}(E)} C_{2}(E)<\infty
$$

for almost all $E \in \mathbb{R}$ with respect to Lebesgue measure. Therefore, by Lemma 5.2 it follows that system (2.9) has no subordinate solutions for almost all $E \in \mathbb{R}$ with respect to Lebesgue measure. To complete the proof, it remains to use Theorem 3.7 .

Proof of Corollary 1.5. We prove that the conditions of Theorem 1.4 are fulfilled. Using Abel's transformation, we can write

$$
\begin{aligned}
\sum_{k=1}^{n-1} \frac{\left|c_{k}\right|}{\sqrt{k}} e^{2 \sqrt{k}} & =\sum_{k=1}^{n-2}\left(\left|c_{k}\right|-\left|c_{k+1}\right|\right) \sum_{m=1}^{k} \frac{1}{\sqrt{m}} e^{2 \sqrt{m}}+\left|c_{n-1}\right| \sum_{k=1}^{n-1} \frac{1}{\sqrt{k}} e^{2 \sqrt{k}} \\
& \leq \sum_{k=1}^{n-2}\left(\left|c_{k}\right|-\left|c_{k+1}\right|\right) e^{2 \sqrt{k+1}}+\left|c_{n-1}\right| e^{2 \sqrt{n}} \\
& \leq e^{\sqrt{2 n}} \sum_{k=1}^{\frac{n}{2}-1}\left(\left|c_{k}\right|-\left|c_{k+1}\right|\right)+e^{2 \sqrt{n-1}} \sum_{k=\frac{n}{2}}^{n-2}\left(\left|c_{k}\right|-\left|c_{k+1}\right|\right)+\left|c_{n-1}\right| e^{2 \sqrt{n}} \\
& \leq e^{\sqrt{2 n}}\left|c_{1}\right|+e^{2 \sqrt{n-1}}\left|c_{\frac{n}{2}}\right|+\left|c_{n-1}\right| e^{2 \sqrt{n}} .
\end{aligned}
$$


Hence,

$$
\sum_{n=1}^{\infty} \frac{\left|c_{n}\right|}{\sqrt{n}} e^{-2 \sqrt{n}} \sum_{k=1}^{n-1} \frac{\left|c_{k}\right|}{\sqrt{k}} e^{2 \sqrt{k}} \leq\left|c_{1}\right| \sum_{n=1}^{\infty} \frac{\left|c_{n}\right|}{\sqrt{n}} e^{-\frac{1}{2} \sqrt{n}}+\sum_{n=1}^{\infty} \frac{\left|c_{n}\right|\left|c_{\frac{n}{2}}\right|}{\sqrt{n}}+\sum_{n=1}^{\infty} \frac{\left|c_{n}\right|\left|c_{n-1}\right|}{\sqrt{n}}
$$

The assumptions of Corollary 1.5 imply that the series on the right-hand side of (6.3) are convergent. Therefore, the series on the left-hand side of (16.3) also converge, so that we can use Theorem 1.4.

Proof of Theorem 1.6. We make the substitution

$$
\mathbf{a}(n)=\exp \left(i \sum_{p=1}^{n-1} \Delta(p) \sigma_{3}\right) \mathbf{c}(n)
$$

in (2.4). The equation for $\mathbf{c}$ takes the form

$$
\mathbf{c}(n+1)=D(n) \mathbf{c}(n),
$$

where

$$
D(n)=\exp \left(-i \sum_{p=1}^{n} \Delta(p) \sigma_{3}\right) T(n) \exp \left(i \sum_{p=1}^{n-1} \Delta(p) \sigma_{3}\right), \quad \operatorname{det} D(n)=1 .
$$

The asymptotic formula (2.7) for the matrix $T(n)$ shows that $D(n)$ admits the following estimate as $n \rightarrow \infty$ :

$$
\|D(n)-I\|=O\left(\frac{\left|c_{n}\right|}{\sqrt{n}}\right)+O\left(d_{n}\right) .
$$

Estimates (1.3) and (6.6) imply that the following ordered product of matrices converges:

$$
\prod_{n \geq 1}^{\curvearrowright} D(n)
$$

Consequently, an arbitrary solution of system (6.5) tends to a constant vector as $n \rightarrow \infty$. Using (6.4), we conclude that, as $n \rightarrow \infty$, an arbitrary solution of system (2.4) admits an asymptotic expansion of the form

$$
\mathbf{a}(n)=\exp \left(i \sum_{p=1}^{n-1} \Delta(p) \sigma_{3}\right)\left(\mathbf{a}_{0}+o(1)\right),
$$

where $\mathbf{a}_{0}$ is a constant vector. Since, obviously, $\|\mathbf{a}(n)\|=\|\mathbf{b}(n)\|$, we easily see that system (2.9) has no subordinate solutions for any $E \in \mathbb{R}$. Thus, Theorem 3.6 implies the first statement of Theorem 1.6.

Now, we substitute the asymptotic expansion (6.7) in (2.3) and use the integral equations (2.5) and (2.6). As a result, we see that, as $x \rightarrow+\infty$, an arbitrary solution $\psi$ of equation (1.4) admits an asymptotic expansion of the form

$$
\begin{aligned}
\psi(x)= & \frac{C_{4}}{\sqrt[4]{n}} \exp \left(i \sum_{p=1}^{n-1} \Delta(p)+i k_{n}(x-n)\right) \\
& +\frac{C_{5}}{\sqrt[4]{n}} \exp \left(-i \sum_{p=1}^{n-1} \Delta(p)+i k_{n}(x-n)\right)+o\left(\frac{\left|C_{4}\right|+\left|C_{5}\right|}{\sqrt[4]{n}}\right), \quad x \in[n, n+1],
\end{aligned}
$$


where $C_{4}$ and $C_{5}$ are constants. To complete the proof, we put $C_{5}=0$ and use the elementary relations

$$
\begin{gathered}
\int_{n}^{n+1}\left[\sqrt{t+E}-\frac{q(t)}{2 \sqrt{t+E}}\right] d t=\Delta(n)+O\left(n^{-3 / 2}\right), \\
\int_{n}^{x}\left[\sqrt{t+E}-\frac{q(t)}{2 \sqrt{t+E}}\right] d t=k_{n}(n-x)+O\left(n^{-1 / 2}\right) .
\end{gathered}
$$

Acknowledgments. The author wants to thank V. S. Buslaev for attention to this work and numerous stimulating discussions.

\section{REFERENCES}

[1] V. S. Buslaev and L. D. Faddeev, Formulas for traces for a singular Sturm-Liouville differential operator, Dokl. Akad. Nauk SSSR 132 (1960), no. 1, 13-16; English transl., Soviet Math. Dokl. 1 (1960), 451-454. MR0120417(22:11171)

[2] S. N. Naboko and A. B. Pushnitskiu, A point spectrum, lying on a continuous spectrum, for weakly perturbed operators of Stark type, Funktsional. Anal. i Prilozhen. 29 (1995), no. 4, 31-44; English transl., Funct. Anal. Appl. 29 (1995), no. 4, 248-257 (1996). MR.1375539 (96k:34187)

[3] A. Zygmund, Trigonometric series. Vols. I, II, third edition, Cambridge Univ. Press, Cambridge, 2002. MR1963498 (2004h:01041)

[4] A. A. Pozharskiǔ, Wannier-Stark type operators with singular potentials, Algebra i Analiz 14 (2002), no. 1, 158-193; English transl., St. Petersburg Math. J. 14 (2003), no. 1, 119-145. MR1893324 (2003e:81051)

[5] - On the spectrum of the Wannier-Stark operator, Algebra i Analiz 16 (2004), no. 3, 171-200; English transl., St. Petersburg Math. J. 16 (2005), no. 3, 561-581. MR2083569 (2005e:81059)

[6] H. L. Cycon, R. G. Froese, W. Kirsch, and B. Simon, Schrödinger operators with application to quantum mechanics and global geometry, Springer-Verlag, Berlin, 1987. MR0883643 (88g:35003)

[7] V. S. Buslaev, Kronig-Penney electron in a homogeneous electric field, Differential Operators and Spectral Theory, Amer. Math. Soc. Transl. (2), vol. 189, Amer. Math. Soc., Providence, RI, 1999, pp. 45-57. MR1730502 (2001b:81039)

[8] M. Christ and A. Kiselev, Absolutely continuous spectrum of Stark operators, www.arxiv.org: math.SP/0110141 v2 17 Oct 2001. MR.1971938(2005a:81066)

[9] P. Deift and R. Killip, On the absolutely continuous spectrum of one-dimensional Schrödinger operators with square summable potentials, Comm. Math. Phys. 203 (1999), 341-347. MR1697600 (2000c:34223)

[10] D. J. Gilbert and D. B. Pearson, On subordinacy and analysis of the spectrum of one-dimensional Schrödinger operators, J. Math. Anal. Appl. 128 (1987), 30-56. MR0915965 (89a:34033)

[11] Y. Last and B. Simon, Eigenfunctions, transfer matrices, and absolutely continuous spectrum of one-dimensional Schrödinger operators, Invent. Math. 135 (1999), 329-367. MR1666767 (2000f:47060)

[12] G. Perelman, On the absolutely continuous spectrum of Stark operators, Comm. Math. Phys. 234 (2003), 359-381. MR.1962465 (2004c:81081)

Department of Mathematics and Mechanics, St. Petersburg State University, UniverSitetskit Prospekt 28, Petrodvorets, St. Petersburg, 198504, Russia

E-mail address: pozharsky@math.nw.ru

Received 31/OCT/2006

Translated by B. M. BEKKER 